\title{
The continuing question of how fever duration is associated with patient outcome
}

\author{
Panagiotis Kiekkas ${ }^{* 1}$, Diamanto Aretha ${ }^{2}$ and George I Baltopoulos ${ }^{3}$ \\ See related research by Seguin et al., http://ccforum.com/content/16/4/R150
}

\begin{abstract}
Although fever magnitude and etiology have been associated with outcomes of critically ill patients, possible associations between fever duration and mortality remain inconclusive. Since long-lasting fever is generally attributed to severe pathologic conditions, it is expected to be an indicator of adverse outcome. It also seems plausible that persistent fever in specific patient groups, mainly those with cerebral damage or limited cardiorespiratory reserve, could lead to worsened outcomes. Existing studies on these associations have been considerably limited because of methodological flaws, which may account for controversial findings that have been reported. Welldesigned, large-sample studies using diverse measures of fever duration need to be conducted.
\end{abstract}

In a recent issue of Critical Care, Seguin and colleagues [1] presented a multicenter study on the incidence, risk factors, and outcomes of prolonged fever in surgical intensive care unit (ICU) patients. The authors' findings provided valuable insight into the etiologies of fever lasting more than 5 days. In this context, prolonged fever may pose suspicion about specific infectious and noninfectious pathologic conditions and therefore contribute to the diagnostic process. However, the question of whether fever duration is associated with mortality remains.

Associations between fever characteristics and patient outcomes have attracted particular interest in recent years. Fever magnitude has been identified as a significant determinant of in-hospital or ICU mortality in surgical

*Correspondence: kiekkpan@otenet.gr

'Nursing Department, Highest Technological Educational Institute of Patras, 1 M.

Alexandrou Str., Patras, 263-34, Greece

Full list of author information is available at the end of the article and general ICU patients, and temperatures exceeding $39.3^{\circ} \mathrm{C}$ to $39.5^{\circ} \mathrm{C}$ have been associated with higher risk for death [2-4]. In regard to fever etiology, a recent study supported the positive effects of temperature increase on survival of patients with sepsis [5], advocating a more permissive antipyretic treatment for them.

Fever of long duration can be an indicator of worsened outcome. Seguin and colleagues [1] identified severe sepsis and cerebral injury as independent risk factors for prolonged fever. However, these pathologic conditions are also among the leading causes of death in adults [6,7] and therefore could be the underlying causes of both prolonged fever and increased mortality.

Persistent fever might further be a mediator of worsened outcome. Fever generally benefits the infected host by inducing the expression of heat shock proteins, which protect host cells and regulate immune responses [8]. However, the febrile response is highly regulated by endogenous antipyretics, which suppress pro-inflammatory cytokine synthesis and control fever magnitude and duration [9]. Activation of these substances implies that high or long-lasting fever may aggravate immune function and be maladaptive, possibly by inhibiting apoptosis of immune cells and perpetuating pro-inflammatory cytokine response [2]. Moreover, elevated temperature exacerbates brain tissue damage and is associated with increased in-hospital mortality in neurology/neurosurgery ICU patients [10]. Likewise, sepsis or cardiopulmonary disorders may decrease tolerance to increased metabolic demands during the febrile response, rendering patients susceptible to severe hemodynamic instability and hypoxic tissue injury [11]. In these patient groups, it seems plausible that the longer the duration of fever, the higher the risk for adverse effects.

On the other hand, could long-lasting fever be beneficial? The febrile response provides adaptive advantages against infections. Thus, keeping febrile temperatures during the infectious process, which is often of long duration, is expected to improve host defenses and outcomes. Schulman and colleagues [12] reported that aggressive fever suppression (acetaminophen administration of 
greater than $38.5^{\circ} \mathrm{C}$ and cooling blanket application of greater than $39.5^{\circ} \mathrm{C}$ ) was followed by considerably higher mortality compared with permissive suppression (the same interventions of greater than $40^{\circ} \mathrm{C}, 15.9 \%$ versus $2.6 \%, P=0.06$ ). All febrile patients who died were septic, and the investigators pointed out that fever suppression decreased the duration rather than the magnitude of febrile episodes.

What is the existing evidence about the association between fever duration and mortality? In contrast to the findings by Seguin and colleagues [1], previous studies conducted in general ICU patients by Circiumaru and colleagues [13] and Peres Bota and colleagues [14] reported that fever lasting more than 5 days was associated with increased ICU mortality (62.5\% versus $29.6 \%$ and $67.2 \%$ versus $22.4 \%$, respectively, $P<0.01$ ). In both studies, infection was documented in the majority of cases with persistent fever, whereas non-infectious fever was of short duration and good outcome. Unfortunately, the conclusions of both these studies and of the recent one by Seguin and colleagues [1] should be seen with caution. First, none of these studies adjusted for differences in mortality risk among patients, while the association between fever duration and mortality was not tested at the multivariate level. Second, a 5-day cutoff point was used for defining prolonged fever in all studies, but no rationale for selecting this cutoff point was provided. Since this cutoff point was arbitrary, considerably different findings about the association between fever duration and mortality cannot be excluded in case other cutoff points had been used.

Treating days with fever as a continuous variable seems more appropriate for addressing the association between fever duration and mortality. In this context, two later studies by Barie and colleagues [2] and Kiekkas and colleagues [4] reported no significant difference in days with fever between ICU survivors and non-survivors. Unfortunately, the first study [2] was limited to surgical ICU patients, thus the findings cannot be generalized to medical or trauma admissions. Similarly, patients with cerebral damage were excluded from the second study [4], which might also be underpowered to detect significant associations between fever duration and outcome.

ICU length of stay may significantly differ among patients, and this should possibly be taken into consideration when comparing fever duration among survivors and non-survivors. Laupland and colleagues [3] calculated fever incidence density, defined as the number of days with fever divided by total ICU admission days. Fever incidence density could be used as a complement to the absolute number of days with fever to allow comparisons independent from ICU length of stay.

Given that fever duration may vary considerably during the daytime, overall hours with fever would possibly be a more precise measure of fever duration. In addition, since fever magnitude has been established as a determinant of mortality, the concept of fever burden might be even more appropriate for predicting patient outcome than fever duration alone. Fever burden is the product of fever duration and magnitude, is estimated as the area under the curve for the time period that the patient manifests fever, and has long been used for comparing the effectiveness of antipyretic treatment [15].

Multicenter cohort studies in the mixed ICU population need to be conducted to elucidate the association between fever duration and mortality by using diverse measures of fever duration and examining whether any of them is independently associated with outcome. If such associations were to be confirmed, experimental studies would be needed to evaluate whether decreasing fever duration with antipyretic treatment could affect patient outcomes.

\section{Abbreviation}

ICU, intensive care unit.

\section{Competing interests}

The authors declare that they have no competing interests.

\section{Author details}

'Nursing Department, Highest Technological Educational Institute of Patras, 1 M. Alexandrou Str., Patras, 263-34, Greece. ${ }^{2}$ Anesthesiology Department, General Pyrgos Hospital, Sintriada, Pyrgos, 271-00, Greece. ${ }^{3}$ Nursing Department, National and Kapodistrian University of Athens, 123 Papadiamantopoulou Str., Athens, 157-73, Greece.

Published: 9 November 2012

\section{References}

1. Seguin P, Roquilly A, Mimoz O, Le Maguet P, Asehnoune K, Biederman S, Carise E, Malledant Y: Risk factors and outcomes for prolonged vs. brief fever - a prospective cohort study. Crit Care 2012, 16:R150.

2. Barie PS, Hydo LJ, Eachempati SR: Causes and consequences of fever complicating critical surgical illness. Surg Infect (Larchmt) 2004, 5:145-159.

3. Laupland KB, Shahpori R, Kirkpatrick AW, Ross T, Gresgon DB, Stelfox HT: Occurrence and outcome of fever in critically ill adults. Crit Care Med 2008, 36:1531-1535.

4. Kiekkas P, Velissaris D, Karanikolas M, Aretha D, Samios A, Skartsani C, Baltopoulos Gl, Filos KS: Peak body temperature predicts mortality in critically ill patients without cerebral damage. Heart Lung 2010, 39:208-216.

5. Fever and Antipyretic in Critically ill patients Evaluation (FACE) Study Group, Lee BH, Inui D, Suh GY, Kim JY, Kwon JY, Park J, Tada K, Tanaka K, letsugu K, Uehara K, Dote K, Tajimi K, Morita K, Matsuo K, Hoshino K, Hosokawa K, Lee KH, Lee KM, Takatori M, Nishimura M, Sanui M, Ito M, Egi M, Honda N, Okayama N, Shime N, Tsuruta R, Nogami S, Yoon SH, Fujitani S, et al:: Association of body temperature and antipyretic treatments with mortality of critically ill patients with and without sepsis: multi-centered prospective observational study. Crit Care 2012, 16:R33.

6. Levinson AT, Casserly BP, Levy MM: Reducing mortality in severe sepsis and septic shock. Semin Respir Crit Care Med 2011, 32:195-205.

7. Feigin VL, Barker-Collo S, Krishnamurthi R, Theadom A, Starkey N: Epidemiology of ischaemic stroke and traumatic brain injury. Best Pract Res Clin Anaesthesiol 2010, 24:485-494

8. Hasday JD, Singh IS: Fever and the heat shock response: distinct, partially overlapping processes. Cell Stress Chaperones 2000, 5:471-480.

9. Tatro JB, Sinha PS: The central melanocortin system and fever. Ann N Y Acad Sci 2003, 994:246-257.

10. Diringer MN, Reaven NL, Funk SE, Uman GC: Elevated body temperature independently contributes to increased length of stay in neurologic intensive care unit patients. Crit Care Med 2004, 32:1489-1495. 
11. Hasday JD, Garrison A: Antipyretic therapy in patients with sepsis. Clin Infect Dis 2000, 31:S234-241.

12. Schulman Cl, Namias N, Doherty J, Manning RJ, Li P, Alhaddad A, Lasko D, Amortegui J, Dy CJ, Dlugasch L, Baracco G, Cohn SM: The effect of antipyretic therapy upon outcomes in critically ill patients: a randomized, prospective study. Surg Infect (Larchmt) 2005, 6:369-375.

13. Circiumaru B, Baldock G, Cohen J: A prospective study of fever in the intensive care unit. Intensive Care Med 1999, 25:668-673.

14. Peres Bota D, Lopes Ferreira F, Melot C, Vincent JL: Body temperature alterations in the critically ill. Intensive Care Med 2004, 30:811-816.
15. Diringer MN, Neurocritical Care Fever Reduction Trial Group: Treatment of fever in the neurologic intensive care unit with a catheter-based heat exchange system. Crit Care Med 2004, 32:559-564.

doi:10.1186/cc11683

Cite this article as: Kiekkas P, et al.: The continuing question of how fever duration is associated with patient outcome. Critical Care 2012, 16:166. 